\title{
CD4+ Tregs and immune control
}

\author{
Zoltán Fehérvari' and Shimon Sakaguchi'1,2,3
}

\begin{abstract}
1Department of Experimental Pathology, Institute for Frontier Medical Sciences, Kyoto University, Kyoto, Japan. 2Laboratory of Immunopathology, Research Center for Allergy and Immunology, The Institute for Physical and Chemical Research (RIKEN), Yokohama, Japan.

${ }^{3}$ Core Research for Evolutional Science and Technology, Japan Science and Technology Agency, Kawaguchi, Japan.
\end{abstract}

\begin{abstract}
Recent years have seen Tregs become a popular subject of immunological research. Abundant experimental data have now confirmed that naturally occurring $\mathrm{CD}^{+} 5^{+} \mathrm{CD}^{+}$Tregs in particular play a key role in the maintenance of self tolerance, with their dysfunction leading to severe or even fatal immunopathology. The sphere of influence of Tregs is now known to extend well beyond just the maintenance of immunological tolerance and to impinge on a host of clinically important areas from cancer to infectious diseases. The identification of specific molecular markers in both human and murine immune systems has enabled the unprecedented investigation of these cells and should prove key to ultimately unlocking their clinical potential.
\end{abstract}

\section{Introduction}

Naturally occurring CD $25^{+} \mathrm{CD} 4^{+}$suppressor or Tregs cells play an active part in establishing and maintaining immunological unresponsiveness to self constituents (i.e., immunological self tolerance) and negative control of various immune responses to non-self antigens (1). Although not a new idea for immunologists, the existence of Tregs as a definite cellular entity has been of great controversy until recently because of the paucity of reliable markers for defining the cell, the ambiguity in the molecular basis of suppressive phenomena, the lack of ample evidence for their roles in immunological disease, and even the elusive nature of some suppressive phenomena themselves (2). Recent years, however, have witnessed increasing interest in Tregs in many fields of basic and clinical immunology. Among the several types of Tregs so far reported, naturally occurring $\mathrm{CD} 25^{+} \mathrm{CD} 4^{+}$Tregs are the main focus of current research, because accumulating evidence indicates that this population plays a crucial role in the maintenance of immunological self tolerance and negative control of pathological as well as physiological immune responses. A prominent feature of $\mathrm{CD}_{25} 5^{+} \mathrm{CD} 4^{+}$Tregs is that the majority, if not all, of them are naturally produced by the normal thymus as a functionally distinct and mature subpopulation of $\mathrm{T}$ cells and persist in the periphery with stable function, and that their generation is, at least in part, developmentally controlled (1). Congenital deficiency of this population, therefore, results in serious impairment of self tolerance and immunoregulation, leading to severe autoimmunity, immunopathology, and allergy in humans (3). On the other hand, their natural presence in the immune system as a phenotypically distinct population makes them a good target for designing ways to treat or prevent immunological diseases and to control pathological as well as physiological immune responses. In addition to this naturally arising "professional" Treg population, there are several other types of Tregs that can be induced from naive $T$ cells by antigenic stimulation under specialized conditions in the periphery $(4,5)$. Although physiological roles for

Nonstandard abbreviations used: GITR, glucocorticoid-induced TNF receptor family-related gene; GITRL, GITR ligand; IBD, inflammatory bowel disease; IDO, indoleamine 2,3-dioxygenase; IPEX, immune dysregulation, polyendocrinopathy, enteropathy, X-linked syndrome; RAG, recombinase-activating gene; T1D, type 1 diabetes; $\operatorname{Tr} 1$, T regulatory cell type 1 .

Conflict of interest: The authors have declared that no conflict of interest exists.

Citation for this article: J. Clin. Invest. 114:1209-1217 (2004).

doi:10.1172/JCI200423395 these inducible or "adaptive" Tregs need to be fully established, they can still be exploited as a therapeutic tool (6). In this article, we shall review recent progress in our understanding of the roles of natural and adaptive $\mathrm{CD}^{+}$Tregs in immune tolerance and negative control of immune responses. We shall also touch briefly on their possible clinical applications.

\section{Naturally occurring CD25+CD4+ Tregs in self tolerance and their production by the normal thymus}

Experimental evidence for the existence of Tregs with autoimmune-inhibitory activity has been suggested in various animal models of autoimmune disease for many years $(7,8)$. Neonatal thymectomy, for example, leads to spontaneous development of autoimmune diseases including gastritis, thyroiditis, and oophoritis in selected strains of mice (7-9). Adult thymectomy and subsequent sublethal $\mathrm{X}$-irradiation produced thyroiditis and type 1 diabetes (T1D) in selected strains of rats $(10,11)$. In NOD mice or Bio-Breeding (BB) rats, which spontaneously develop T1D and autoimmune thyroiditis, inoculation with $\mathrm{CD}^{+} \mathrm{T}$ cells from histocompatible normal animals effectively prevented T1D $(12,13)$. On the other hand, characterization of effector $\mathrm{T}$ cells mediating these organ-specific autoimmune diseases firmly documented that $\mathrm{CD}^{+}$Th cells destroy the target organs/tissues by helping B cells to form specific autoantibodies and by inducing cell-mediated immune responses to the target self antigens. Collectively, these findings suggested that normal individuals harbor 2 functionally distinct populations of $\mathrm{CD}^{+} \mathrm{T}$ cells, one capable of mediating autoimmune disease and the other capable of dominantly inhibiting it in the normal physiological state (8). To test this hypothesis directly, attempts were made from the mid-1980s onward to dissect these $2 \mathrm{CD}^{+} \mathrm{T}$ cell populations by expression levels of particular cell surface molecules and to examine their potential correlation with autoimmune induction or inhibition. When $\mathrm{CD}^{+}$ splenic $T$ cell suspensions prepared from normal mice or rats were depleted of $\mathrm{CD}_{25} 5^{+}, \mathrm{RT} 6.1^{+}, \mathrm{CD} 5^{\text {high }}$, or CD $45 \mathrm{RB} / \mathrm{RC}^{\text {low }}$ cells and the remaining $\mathrm{CD}^{+} \mathrm{T}$ cells transferred to syngeneic $\mathrm{T}$ cell-deficient mice or rats, the recipients spontaneously developed various organ-specific autoimmune diseases (including T1D, thyroiditis, and gastritis) and systemic wasting disease in a few months; reconstitution of the eliminated population inhibited the development of autoimmune disease $(1,8,14)$. A similar transfer experiment also induced inflammatory bowel disease (IBD), which appeared to result from an excessive immune response of $\mathrm{T}$ cells to com- 


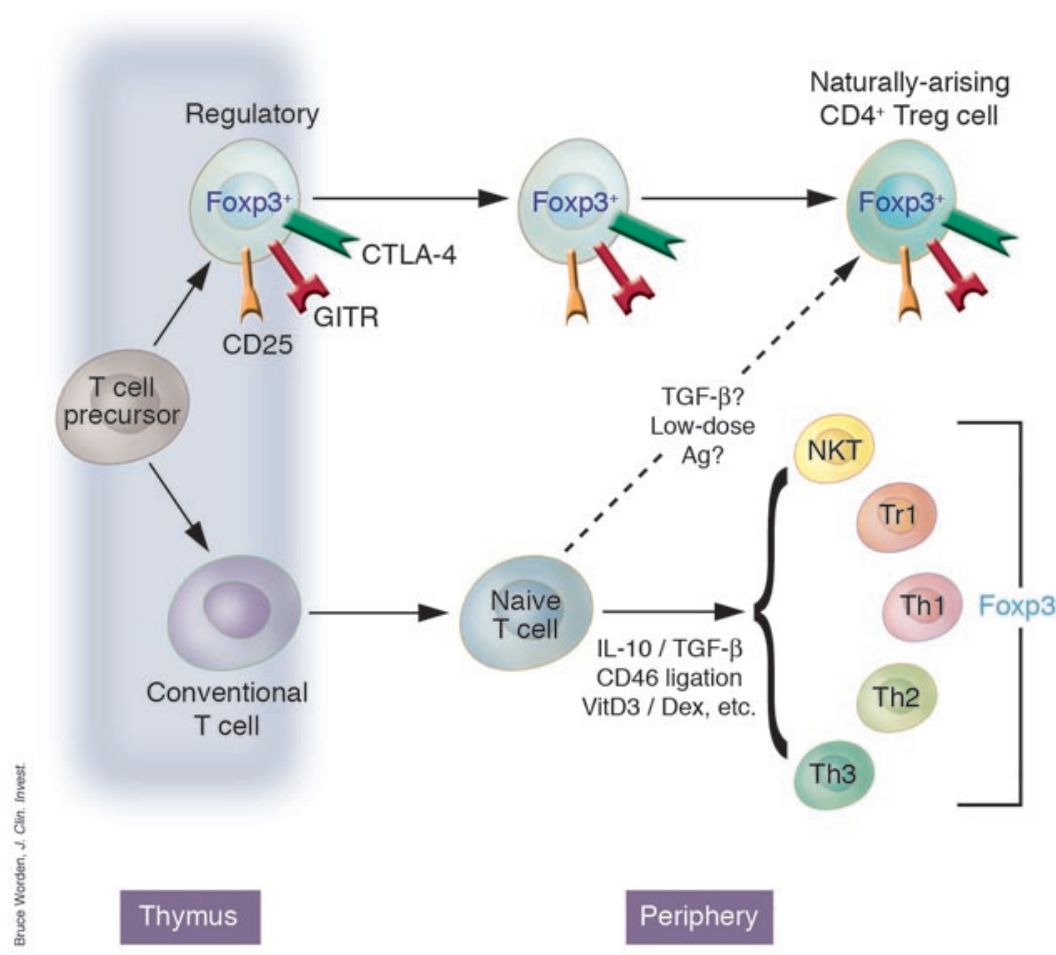

\section{Figure 1}

Regulatory $\mathrm{CD}^{+}$cells can develop in a number of ways, although the mechanisms by which these occur and the relationship of the resulting cells to one another are contestable. Thymically generated Treg cells, otherwise known as natural $T_{R}$ cells or $C D 25^{+} C D 4^{+} T_{R}$ cells, develop intrathymically according to a specialized combination of TCR and costimulatory signals. Extrathymically generated $T_{R}$ cells, e.g., Tr1 cells or Th3 cells, can be generated under a whole host of conditions. Whether a conventional naive CD4+ T cell can be converted in the periphery to a de facto Foxp3 ${ }^{+}$ $T_{R}$ cell remains controversial. mensal bacteria in the intestine (15). Currently CD25 is the most specific cell surface marker for such autoimmune- and IBD-preventive $\mathrm{CD} 4^{+} \mathrm{T}$ cells, because $\mathrm{CD} 25^{+} \mathrm{CD} 4^{+} \mathrm{T}$ cells, which constitute $5-10 \%$ of $\mathrm{CD}^{+} \mathrm{T}$ cells in normal naive mice, are included in the $\mathrm{CD} 5^{\text {high }}$ or $\mathrm{CD} 45 \mathrm{RB}^{\text {low }} \mathrm{CD} 4^{+}$population, and furthermore their depletion alone is sufficient to cause autoimmune disease/IBD, while their reconstitution is effectively able to inhibit autoimmune disease/IBD, in various models $(1,16)$. Additionally, the various immunological properties of natural Tregs, including their in vitro suppressive activity, are assigned to $\mathrm{CD} 25^{+} \mathrm{CD} 4^{+} \mathrm{T}$ cells naturally arising in the immune system $(1,8,14)$. It should be noted, however, that CD25 is not an absolute marker for naturally occurring Tregs, since it is also expressed at high levels on activated but otherwise conventional nonregulatory $\mathrm{T}$ cells. We shall discuss this caveat later in the article.

The normal thymus produces the majority, if not all, of $\mathrm{CD} 25^{+} \mathrm{CD} 4^{+}$Tregs as a functionally mature $\mathrm{T}$ cell subpopulation, which appears to constitute a distinct cellular lineage and to be contiguous with those found in the periphery (17) (Figure 1). As shown with the transfer of $\mathrm{CD} 25^{-} \mathrm{CD} 4^{+}$spleen cells described above, transfer of mature thymocyte suspensions depleted of $\mathrm{CD} 25^{+}$thymocytes produced various autoimmune diseases in syngeneic T cell-deficient mice (17). This indicates that the normal thymus is continuously producing pathogenic self-reactive $\mathrm{CD}^{+}$ $\mathrm{T}$ cells as well as functionally mature $\mathrm{CD} 25^{+} \mathrm{CD} 4^{+}$Tregs capable of controlling them. This centralized production of Tregs has been referred to as "the third function of the thymus" (18).

Accumulating evidence also indicates that thymic development of $\mathrm{CD} 25^{+} \mathrm{CD} 4^{+}$Tregs requires unique interactions of their TCR with self-peptide/MHC complexes expressed by thymic stromal cells (19). In TCR transgenic mice, for example, a large number of $\mathrm{CD} 25^{+} \mathrm{CD} 4^{+} \mathrm{T}$ cells express endogenous TCR $\alpha$ chains paired with transgenic $\beta$ chains; recombinase-activating gene- 2 (RAG-2) defi- ciency, which blocks the gene rearrangement of the endogenous TCR $\alpha$ chain locus, abrogates the development of CD25 $5^{+} \mathrm{CD} 4^{+}$ Tregs in such TCR transgenic mice $(17,20)$. Furthermore, compared with thymic selection of other T cells, the development of $\mathrm{CD} 25^{+} \mathrm{CD} 4^{+}$Tregs requires higher-avidity interactions of their TCRs with self peptide/MHC or class II MHC itself expressed on the thymic stromal cells (especially cortical epithelial cells), yet the required avidity must not be so high as to lead to their deletion (19-23). Accessory molecules, such as CD28, B7, and CD40, expressed on developing thymocytes and thymic stromal cells also contribute to the thymic generation of $\mathrm{CD} 25^{+} \mathrm{CD} 4^{+}$Tregs $(24,25)$.

\section{The naturally occurring CD4+ Treg phenotype}

Naturally occurring $\mathrm{CD} 4^{+}$Tregs constitutively express a variety of cell surface molecules more commonly associated with activated/ memory cells, most significantly CD25, CD45RB low, CD62L, CD103, cytotoxic T lymphocyte antigen-4 (CTLA-4, or CD152), and glucocorticoid-induced TNF receptor family-related gene (GITR) $(15,16$, 26-30). Neuropilin-1, a molecule more usually associated with axon guidance, was very recently reported to be constitutively expressed by natural Tregs and, interestingly, is downregulated on conventional T cells following activation (31). Even though none of these markers is uniquely expressed by naturally occurring CD4+ Tregs, their level of expression and constitutive nature have still made them useful as functional descriptors by enabling the consistent isolation and investigation of $\mathrm{CD}^{+} \mathrm{T}$ cells with regulatory properties. The naturally occurring Treg surface phenotype indicates that they are in an antigen-primed state and are, at least superficially, similar to memory-type $\mathrm{T}$ cells. Judging from the finding that $\mathrm{CD} 25^{+} \mathrm{CD} 4^{+}$Tregs require a high-avidity interaction with self peptide/MHC for their thymic development and become functional within the thymus, one could speculate that they are broad in antigen specificity yet more capable of recognizing self antigens than other $\mathrm{T}$ cells are. 
Although CD25 has so far proven to be the best surface marker for thymically produced $\mathrm{CD}^{+}$Tregs, it can be expressed on any $\mathrm{T}$ cell following activation (1). In the human system, where there are relatively large numbers of activated $T$ cells, this is especially problematic. Currently, therefore, the best way to select natural human $\mathrm{CD}^{+}$Tregs is to sort the population that is very high in CD25 (32). The high constitutive expression of CD25 by Tregs begs the question of whether it is simply a convenient marker or a molecule essential for their function. Several lines of evidence indicate that $\mathrm{CD} 25$ is indispensable for the maintenance of natural CD $25^{+} \mathrm{CD} 4^{+}$ Tregs in the immune system. For example, it has been shown that mice deficient in IL-2, IL-2R $\alpha$ (CD25), or IL-2R $\beta$ (CD122) develop lethal inflammatory disease, termed IL-2 deficiency syndrome, which can be prevented by inoculation of normal CD $25^{+} \mathrm{CD} 4^{+} \mathrm{T}$ cells as long as a source of IL-2 is made available experimentally (33-35). Our own experiments indicated that neutralization of IL-2 selectively reduced numbers of $\mathrm{CD} 25^{+} \mathrm{CD} 4^{+} \mathrm{T}$ cells in normal mice and consequently produced organ-specific autoimmune diseases similar to those produced by depletion of natural Tregs ( $R$. Setoguchi et al., manuscript submitted for publication). Collectively, these results suggest that IL-2 is essential for the development, maintenance, and function of $\mathrm{CD} 25^{+} \mathrm{CD} 4^{+}$Tregs.

GITR and its role in $\mathrm{CD} 25^{+} \mathrm{CD} 4^{+}$Treg function is an interesting area. This molecule was identified as a constitutively expressed marker for naturally occurring Tregs, but, like most such candidate molecules involved in Treg identification, it is also upregulated on conventional activated $\mathrm{CD}^{+}{ }^{+} \mathrm{T}$ cells $(29,30)$. An anti-mouse GITR mAb (DTA-1) is able to block $\mathrm{CD} 25^{+} \mathrm{CD} 4^{+}$Treg suppression in vitro, and, furthermore, its injection leads to the induction of autoimmunity in vivo as well as enhancing the proliferation of CD25- ${ }^{-} D 4^{+}$cells by transducing a costimulatory signal $(29,36)$. Since DTA-1 is nondepleting, it was originally presumed to primarily transmit a suppression-blocking signal to the $\mathrm{CD} 25^{+} \mathrm{CD} 4^{+}$ Treg. However, some very recent data instead suggest that ligation of GITR on activated T cells, not Tregs, renders them resistant to suppression (37). The natural ligand for GITR (GITRL) has now also been cloned and its distribution elucidated (38). GITRL is expressed on APCs (DCs, macrophages, and B cells) but is downregulated following maturation. Therefore the relative distribution patterns of GITR on activated T cells and Tregs, and of GITRL on APCs, suggest a complex dynamic of interaction, which is only just being elucidated.

Identification of an unambiguous surface marker for naturally occurring CD4 ${ }^{+}$Tregs remains something of a Holy Grail, especially where the isolation of human Tregs for clinical purposes is concerned. Efforts in this direction may well guide the progress of research in naturally occurring human Tregs.

\section{FOXP3 as a master control gene for Treg development}

A deeper understanding of the developmental processes of natural Tregs, as suggested by the neonatal thymectomy model of autoimmune disease, evolved out of studies on the Scurfy mouse and the human disease IPEX (immune dysregulation, polyendocrinopathy, enteropathy, X-linked syndrome). IPEX is an $\mathrm{X}$-linked immunodeficiency syndrome associated with autoimmune disease in multiple endocrine organs (such as T1D and thyroiditis), IBD, atopic dermatitis, and fatal infections (3). The Scurfy mouse strain exhibits a fatal X-linked lymphoproliferation characterized by a multiorgan immunopathology very similar to the human disease IPEX (39-41). The causative gene, Foxp3
(FOXP3 in humans), which underlies both syndromes, encodes a forkhead/winged-helix family transcriptional repressor called Scurfin (42-44). The striking similarities seen between mutations in Foxp3/FOXP3 and depletion of $\mathrm{CD} 25^{+} \mathrm{CD} 4{ }^{+}$Tregs led several groups to investigate the relationship of this gene to Treg development and function. Experiments in mice indeed demonstrated Foxp3 mRNA and Scurfin protein to be specifically expressed in $\mathrm{CD} 25^{+} \mathrm{CD} 4^{+}$Tregs, and, in contrast to the cell surface markers used to date, they were never observed in non-Tregs following conventional activation or differentiation into Th1 and Th2, nor in natural killer T cells (45-47).Subsequent studies, also in mice, have further demonstrated the existence of a small population of $\mathrm{CD} 25^{-} \mathrm{CD} 4^{+} \mathrm{T}$ cells that are nevertheless still Foxp $3^{+}$and have a regulatory function (ref. 1 , and $\mathrm{M}$. Ono et al., manuscript submitted for publication). Scurfy mutant mice, or those with a targeted deletion of Foxp3, were unable to support the development of natural $\mathrm{CD} 25^{+} \mathrm{CD} 4^{+}$Tregs, although they contained large numbers of chronically activated $\mathrm{CD} 25^{+}$nonregulatory $\mathrm{T}$ cells $(45,47)$. By contrast, the number of $\mathrm{CD} 25^{+} \mathrm{CD} 4^{+}$Tregs increased significantly in transgenic mice overexpressing Foxp3 (45). A final critical observation showed that retroviral transduction of Foxp3 into Foxp $3^{-}$nonregulatory CD25- $\mathrm{CD}^{+} \mathrm{T}$ cells bestowed on them a fully functional Treg phenotype; e.g., cotransfer of Foxp3transduced $\mathrm{T}$ cells with CD25-CD4 ${ }^{+} \mathrm{T}$ cells prevented autoimmune disease and IBD in SCID mice (see above) $(46,47)$.

Broadly speaking, an equivalent pattern of FOXP3 expression has now also been reported in human cells, with Treg-like properties being similarly transferable by retroviral transduction (48-51). Already, however, some discrepancies are beginning to emerge between the behavior of human and that of mouse FOXP3/Foxp3 expression. For instance, there is at least 1 example of FOXP3 being apparently induced following standard antibodymediated activation of normal CD25- human T cells; this has not been observed thus far in the murine model (50). Similarly, some instances of CD25- human T cell activation by DCs have also resulted in FOXP3 upregulation (refs. 52, 53, and see below). The possibility remains, however, that the induction of FOXP3 expression in human CD25- cells may simply be a result of the expansion of the human counterpart to the murine $\mathrm{CD} 25^{-} \mathrm{CD} 4^{+}$ Foxp $3^{+}$population described above, as these studies all isolated their Tregs solely on the basis of CD25 (51).

Thus, Foxp3/FOXP3 appears to be a master control gene for the development and function of natural $\mathrm{CD} 25^{+} \mathrm{CD} 4{ }^{+}$Tregs. Given that humans bear natural $\mathrm{CD} 25^{+} \mathrm{CD} 4^{+}$Tregs with a phenotype and function comparable to those found in rodents (32), it is most likely that in IPEX, disruption of the FOXP3 gene abrogates the development of thymic Tregs, leading to hyperactivation of T cells reactive with self antigens, commensal bacteria in the intestine, or innocuous environmental substances, and thus causing autoimmune polyendocrinopathy, IBD, or allergy, respectively. This has several implications for self tolerance and autoimmune/inflammatory disease in humans. First, this is so far the clearest example that an abnormality in naturally arising Tregs is a primary cause of human autoimmune disease, IBD, and allergy. Second, the development of natural Tregs is, at least in part, genetically and developmentally programmed. Third, hemizygous defects of the FOXP3 gene in females illustrate that the mechanism of dominant self tolerance is physiologically operating in humans. Owing to random inactivation of the $\mathrm{X}$ chromosome during lyonization of individual Tregs, hemizygous females have FOXP3-defective Tregs 
and FOXP3-normal ones as a genetic mosaic, yet they are nevertheless completely normal (54). This observation demonstrates that even reduced numbers of Foxp $3^{+}$Tregs are able to dominantly control pathogenic $T$ cells, and, further, that even a partial restoration of Tregs could be sufficient to cure IPEX or, indeed, other autoimmune pathologies. Mechanistic data on Foxp3 are thus far lacking; it is therefore currently unclear how it exerts its effects at a molecular level. The molecular interactions of Foxp3, and indeed the signals triggering its expression, are now an intensely investigated area, and unraveling them may well prove critical to exploiting natural Tregs in a therapeutic setting.

\section{Functional characteristics of natural CD25+CD4+ Tregs and their mechanisms of suppression}

Without question the most remarkable feature of $\mathrm{CD} 25^{+} \mathrm{CD} 4^{+}$ Tregs is their ability to dampen immune responses. They appear capable of suppressing a wide variety of immune cells, encompassing those of both the innate (55-57) and the adaptive immune systems (58-60). This suppressive ability can be modeled in vitro by mixing of titrated numbers of highly purified $\mathrm{CD} 25^{+} \mathrm{CD} 4^{+}$ Tregs and responder cells, typically CD25-CD $4^{+} \mathrm{T}$ cells plus a $\mathrm{T}$ cell stimulus. Under such conditions, the $\mathrm{CD} 25^{+}$population suppresses both the proliferation and, more fundamentally, the IL-2 production of the CD25- cells in a dose-dependent manner (58, $59,61) . \mathrm{CD}_{25} \mathrm{CD}^{+}$Tregs themselves require TCR stimulation, and, it now seems, IL-2, to actually trigger their suppressive effects, but once this condition has been satisfied their ensuing suppression can act non-antigen-specifically $(58,59,61)$. Therefore, suppression is an active process and can be directed against bystander cells. Curiously, $\mathrm{CD} 25^{+} \mathrm{CD} 4^{+}$Tregs themselves are anergic in vitro, i.e., they do not proliferate or produce IL-2 in response to conventional $\mathrm{T}$ cell stimuli such as plate-or bead-bound anti-CD3, concanavlin A (ConA), or splenic APCs. This anergy can, however, be broken by a sufficiently potent stimulus, e.g., the addition of high-dose exogenous IL-2 or anti-CD28, or the use of mature DCs as $\operatorname{APCs}(27,58,59,61-63)$. Some of these strong stimuli, particularly mature DCs, also perturb $\mathrm{CD} 25^{+} \mathrm{CD} 4^{+}$Treg suppression both in vitro and in vivo $(63,64)$. At least in vitro, anergy seems to be the default state of naturally occurring Tregs, since they revert back to it once potent stimulation is withdrawn (58). In vivo, however, $\mathrm{CD} 25^{+} \mathrm{CD} 4^{+}$Treg anergy is not readily observed; instead they seem to have a highly active rate of turnover $(33,65)$. It seems likely, then, that $\mathrm{CD} 25^{+} \mathrm{CD} 4^{+}$Treg anergy is an in vitro phenomenon, merely reflecting an exacting set of activation requirements generally absent from cell culture.

Given that the ability to control immune responses is the cardinal feature of $\mathrm{CD} 25^{+} \mathrm{CD} 4^{+}$Tregs, it is surprising that their mechanism(s) of suppression remains elusive. Essentially, Treg suppression can be divided into those mechanisms mediated by relatively far-reaching soluble factors and those requiring intimate cell contact. In vivo experiments based chiefly on the IBD model mentioned previously have demonstrated the importance of the immunomodulatory cytokines IL-10 and TGF- $\beta$ (66). By blocking IL-10 signaling in vivo with an anti-IL-10 receptor $\mathrm{mAb}$, it was possible to abrogate the normal colitis-preventative action of CD45RB ${ }^{\text {low }}$ cells (66). Similarly, CD45RB low $\mathrm{T}$ cells from IL-10 $0^{-/-}$mice lacked their otherwise intrinsic ability to protect from colitis and, moreover, were even colitogenic themselves when transferred alone (66). The importance of IL-10 is further underscored by the observation that IL-10 $10^{-/}$mice spontaneously develop colitis $(67,68)$. Examination of the in vivo role of TGF- $\beta$ has generally painted a similar picture to that of IL-10, with Treg function being blocked by the presence of neutralizing anti-TGF- $\beta$ mAbs (69). Some data also suggest that TGF- $\beta$ may not necessarily act as a soluble factor but can also be found on the surface of activated $\mathrm{CD} 25^{+} \mathrm{CD} 4^{+}$Tregs and may therefore act in a membrane-proximal manner (70). Interestingly, virtually all TGF- $\beta^{+}$ $\mathrm{CD} 25^{+} \mathrm{CD} 4^{+}$Tregs also express thrombospondin, a factor capable of converting normally latent TGF- $\beta$ into its active form (71). There should be a note of caution regarding these in vitro studies on TGF- $\beta$, since a comprehensive analysis by a second group failed to demonstrate any role for it in vitro (72).

The confusion over a definitive $\mathrm{CD} 25^{+} \mathrm{CD} 4^{+}$Treg suppression mechanism is compounded when viewed in the context of the in vitro data, since here the overwhelming evidence highlights direct cell-cell interaction, and not cytokines, as being critical $(58,59,73)$. Several lines of evidence lead to this conclusion: with the exception of the study on membrane-bound TGF- $\beta$ alluded to above, both anti-IL-10 and anti-TGF- $\beta$ fail to perturb CD $25^{+} \mathrm{CD} 4^{+}$Treg suppression $(58,59,70,72)$. Similarly, supernatants from suppressed cultures or activated $\mathrm{CD} 25^{+} \mathrm{CD} 4^{+}$Tregs show no inherent suppressive activity, nor can suppression be observed across a semipermeable membrane $(58,59)$. Collectively, these in vitro observations therefore appear to obviate a role not just for IL- 10 and TGF- $\beta$ but for soluble factors in general.

The actual membrane events occurring during suppression that depends on $\mathrm{CD} 25^{+} \mathrm{CD} 4^{+}$Treg contact have yet to be clarified. The most simplistic models propose competition for APCs and specific MHC/peptide antigenic complexes. Additionally, the constitutive expression of the high-affinity IL-2 receptor could make naturally occurring Tregs into an effective IL-2 sink, depriving potential autoreactive $\mathrm{T}$ cells of this essential growth factor (74). However, given the relative physiological scarcity of naturally occurring Tregs, it is perhaps unlikely that a simple competitive-adsorptive model alone could account for their suppressive action in vivo. Other models of $\mathrm{CD} 25^{+} \mathrm{CD} 4^{+}$Treg suppression propose a more proactive and antagonistic form of suppression that relies on the expression of specific "inhibitory" molecules. The identity and indeed even the very existence of such an inhibitory molecule are uncertain, but 1 potential molecule could be Treg-expressed CTLA-4. Aside from its well-established high affinity for the costimulatory molecules B7.1 and B7.2 (CD80 and CD86, respectively), CTLA-4 has also recently been shown to trigger the induction of the enzyme indoleamine 2,3-dioxygenase (IDO) when interacting with its ligands on DCs (75-78). IDO catalyzes the conversion of tryptophan to kynurenine and other metabolites, which have potent immunosuppressive effects in the local environment of the DC. In this way, CD $25^{+} \mathrm{CD} 4^{+}$Tregs may exert their suppression by proxy through their action on APCs. Another APC-centric mode of suppression could be via the perturbation of antigen-presenting capacity. In support of this concept, one report has demonstrated that purified $\mathrm{CD} 25^{+} \mathrm{CD} 4^{+}$Tregs are able to downregulate the expression of both CD80 and CD86 on DCs, converting them into inefficient APCs (57). At any rate, $\mathrm{CD} 25^{+} \mathrm{CD} 4^{+}$Tregs need not act exclusively via the APC, since they are quite capable of suppressing in the context of "APC-free" systems such as plate- or bead-bound antibodies or MHC/peptide tetramers $(58,79)$. At least in vitro, direct suppression of the target cell is therefore still also possible.

A provocative investigation into the membrane events involved in $\mathrm{CD} 25^{+} \mathrm{CD} 4^{+}$Treg suppression was recently reported (80). This 


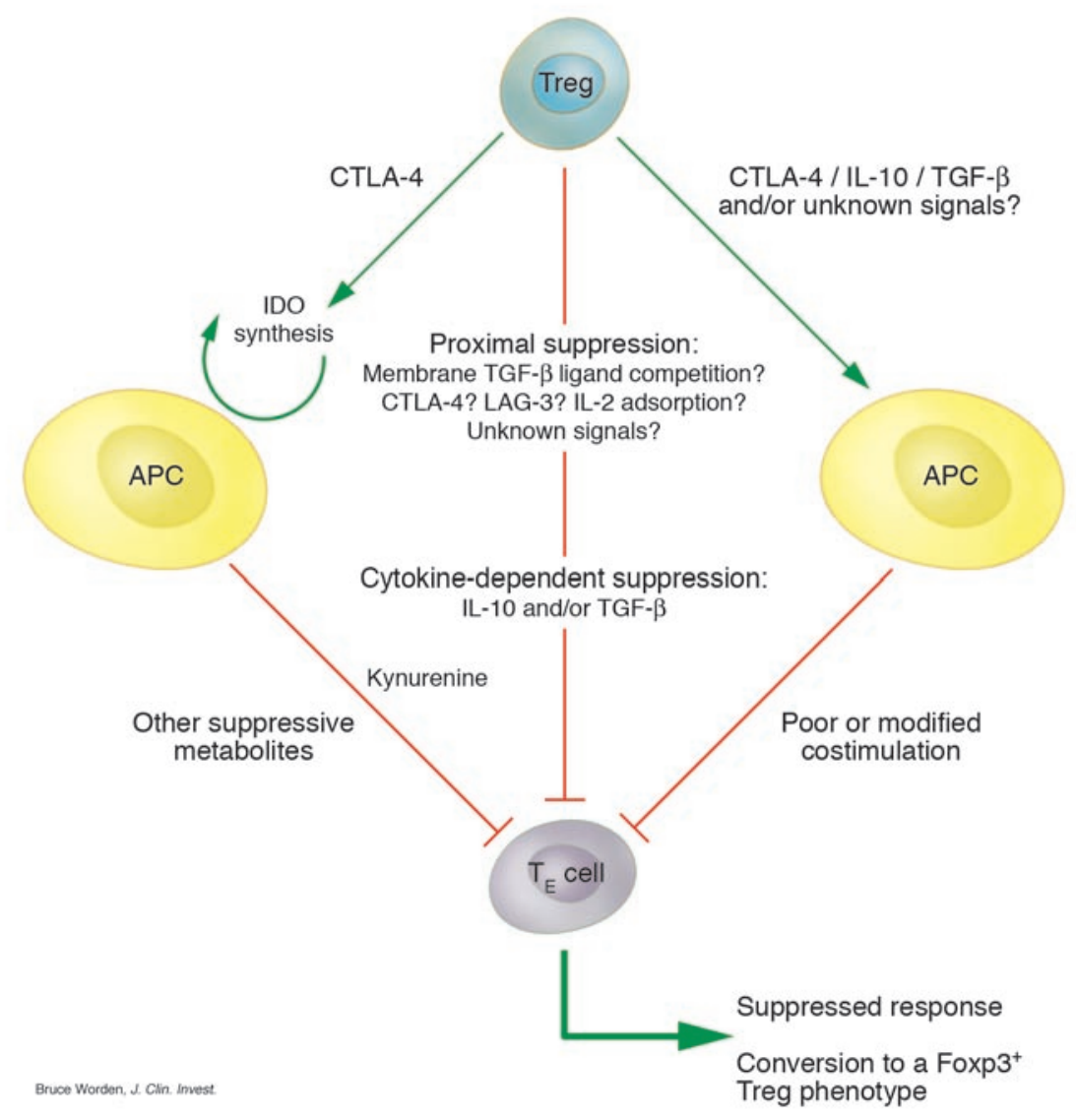

\section{Figure 2}

Possible CD25+CD4+ Treg suppression mechanisms in vivo. CD25+CD4+ Tregs may suppress their effector $T$ cell targets $\left(T_{E}\right)$ by a number of proposed mechanisms. In vivo CD25+CD4+ Tregs may act in a cell contact-dependent manner by competing directly for stimulatory ligands on the APC, by sinking essential growth factors such as IL-2, or by directly transmitting an as-yet uncharacterized negative signal. Alternatively, they may use longerrange suppressive mechanisms by means of the cytokines IL-10 and TGF- $\beta$. Finally, CD $25^{+} \mathrm{CD} 4^{+}$ Tregs may act through the APC either by triggering IDO activity, resulting in the generation of immunosuppressive metabolites, or by perturbing the APC's presenting capacity. Such mechanisms are not necessarily mutually exclusive, and more than 1 might operate in tandem. study suggested that engagement of CD80, and to a lesser extent $\mathrm{CD} 86$, on the responder $\mathrm{T}$ cell and not the APC was responsible for the transmission of a negative signal, and therefore these were the molecular targets through which Tregs exert their function (80). In support of this, the authors demonstrated that B7-/- responder cells were resistant to suppression in vitro and induced a fatal wasting disease refractory to cotransferred $\mathrm{CD} 25^{+} \mathrm{CD} 4^{+}$Tregs (80). Again the obvious candidate Treg molecule for this inhibitory interaction would be CTLA-4, although this fails to explain the paradoxically intact suppression mediated by CTLA-4-/$\mathrm{CD} 25^{+} \mathrm{CD} 4^{+}$Tregs (27). The presence of $\mathrm{B} 7$ on conventional T cells has been known for several years, and it would be interesting, then, if this hitherto puzzling expression pattern were shown to play a role in Treg-mediated suppression $(81,82)$. While the identification of a membrane-bound $\mathrm{CD} 25^{+} \mathrm{CD} 4^{+}$Treg-specific inhibitory molecule remains inconclusive, some very recent work has suggested that the CD4-related molecule LAG-3 may be important (CD223), though this awaits independent confirmation $(83,84)$. Proving a negative hypothesis is always a difficult task, but it may yet be shown that there are no truly unique Treg-associated molecules responsible for inhibition. Rather, the specialized functions of Tregs could simply be the product of known molecules acting semi-redundantly, which together generate a suppressive phenotype. An integrated summary of $\mathrm{CD} 25^{+} \mathrm{CD} 4^{+}$Treg suppressive mechanisms is shown in Figure 2.

Given the relative physiological scarcity of $\mathrm{CD} 25^{+} \mathrm{CD} 4^{+}$Tregs, it seems likely that in vivo they would use mechanisms to amplify their suppressive action, and ones that are not normally fully appreciated under in vitro analysis. This could occur either by the modification of APCs as outlined above or by the "infectious" spreading of tolerance to conventional $\mathrm{T}$ cells. In accordance with this, some recent work has demonstrated that human $\mathrm{CD} 25^{+} \mathrm{CD} 4^{+}$Tregs can confer a suppressive phenotype to conventional $\mathrm{CD}^{+} \mathrm{T}$ cells in a contactdependent manner (52). These newly generated regulatory-like cells then suppress by means of IL- 10 or TGF- $\beta$. This would constitute a mechanism of not only spreading a suppressive phenotype but also making it more efficient on a per-cell basis by engaging the action of soluble mediators. Most satisfyingly, this scenario could also finally reconcile some of the disparities observed between the in vitro and the in vivo mechanisms of $\mathrm{CD} 25^{+} \mathrm{CD} 4^{+}$Treg suppression.

\section{Extrathymic generation of CD4+ Tregs}

The possibility of extrathymic $\mathrm{CD} 25^{+} \mathrm{CD} 4^{+}$Treg generation is currently contentious. An interesting recent study in this area readdressed so-called "low-zone" tolerance (85). This phenomenon was first observed decades ago and was described as the antigen-specific tolerance resulting from subimmunogenic doses of antigen given i.v. (86). Using an osmotic pump to deliver minute controlled quantities of antigenic peptide to TCR transgenic mice, the authors of this updated study demonstrated the appearance of $\mathrm{CD} 25^{+} \mathrm{CD} 4^{+}$Tregs measurable by their function, surface phenotype, and Foxp3 expression. Importantly, the study showed that Treg development could still occur in thymectomized TCR transgenic mice on a RAG knockout background, which are normally wholly lacking in $\mathrm{CD} 25^{+} \mathrm{CD} 4^{+}$Tregs $(17,20$, $46,85)$. It therefore seems possible that $\mathrm{CD} 25^{+} \mathrm{CD} 4^{+}$Treg development, as measured by Foxp3 and suppressive function, can occur in conventional $\mathrm{T}$ cells under specific in vivo activation 


\section{Table 1}

Potential clinical applications of CD25+CD4+ Tregs

\author{
Enhancement of $\mathrm{CD} 25+\mathrm{CD} 4+$ Treg function
}

\author{
Reduction of $\mathrm{CD} 25+\mathrm{CD} 4+$ Treg function
}

Target condition

Organ transplantation, autoimmune disease, allergy

Cancer, infectious disease

\section{Potential therapeutic approach}

Transfer of Tregs or enhancement of their function allows specific suppression of immune responses; e.g., ex vivo gene transduction of Foxp3; ex vivo generation of regulatory cells using cytokines, pharmacological agents, or modified DCs

Removal of Tregs or blocking of their function boosts immune responses; anti-CTLA-4, -CD25, -GITR mAbs conditions, therefore demonstrating a potentially clinically significant developmental plasticity.

The involvement of TGF- $\beta$ in many facets of Treg behavior is well known (87), but a recent report has also suggested that its exogenous addition results in development of Foxp $3^{+}$Tregs, from conventional and even from RAG knockout CD4 ${ }^{+} \mathrm{T}$ cells (refs. 87, 88). However, it remains to be seen whether generation of CD $25^{+} \mathrm{CD} 4^{+}$ Tregs by the mechanisms described above can occur outside of the relatively artificial confines of a TCR transgenic system, and indeed whether the initial cell populations contain only "truly" naive $\mathrm{T}$ cells and no potential Foxp $3^{+} \mathrm{CD} 25^{+} \mathrm{CD} 4^{+}$Treg precursors.

In the original demonstration of extrathymically generated regulatory cells, the cells were termed T regulatory cell type $1(\operatorname{Tr} 1)$ or Th3 cells $(89,90)$. It is likely that these cells form a cell type distinct from their thymically generated $\mathrm{CD} 25^{+} \mathrm{CD} 4^{+}$Treg counterparts that have been elaborated on above. $\operatorname{Tr} 1$ and related cells have been generated using a variety of approaches, typically involving $\mathrm{T}$ cell activation in the presence of immunomodulating cytokines or repetitive stimulation with nonprofessional APCs. $\operatorname{Tr} 1$ cells were initially generated by chronic stimulation of normal nonregulatory $\mathrm{T}$ cells in the presence of IL-10 (89). Such cells secrete a pattern of cytokines distinct from that of the more usual Th1 or Th2 profile and are characterized by high levels of IL-10 and generally low levels of TGF- $\beta$ and IL-5 (89). Moreover, Tr1 cells are functionally suppressive in vivo and able to prevent the development of Th1 autoimmune diseases such as colitis $(89,91)$. Th3 cells, on the other hand, were cloned from the mesenteric lymph nodes of mice orally tolerized with myelin basic protein (90). The majority of such cells produce TGF- $\beta$ and varying levels of Th 2 cytokines and suppress the induction of experimental autoimmune encephalitis (90). In vitro treatment of human and mouse T cells with a combination of the immunosuppressants vitamin $\mathrm{D}_{3}$ and dexamethasone has also resulted in the generation of Foxp $3^{-}$regulatory cells, but with properties somewhat distinct from those reported for $\operatorname{Tr} 1$ or Th3 cells (92-94). Finally, there is also a study suggesting that signaling through the complement receptor (CD46) concomitant to more conventional TCR activation can trigger the peripheral induction of human $\mathrm{CD}^{+}$regulatory cells (95).

Much attention has also focused on the influence that DCs may have on the extrathymic development of regulatory cells. Stimulation with immature DCs (i.e., low levels of costimulatory molecules) and stimulation with DCs modified by pretreatment with IL-10 or TGF- $\beta$ have both been shown to result in the induction of anergic cells with suppressive capabilities in vitro and in vivo (96-98). Current models of DC-based tolerance state that $\mathrm{T}$ cell antigen recognition on immature DCs results in tolerization whereas mature DCs elicit effector responses (99). A system structured in this way would be effective at maintaining self tolerance in the physiological steady state, i.e., in the absence of inflammatory "danger signals," yet would support productive immune responses following DC maturation triggered by the presence of microbes. However, there would always be the potential danger that DCs matured during "sterile" inflammation, e.g., following mechanical injury, could elicit immunity to autoantigens. Similarly, self tolerance could theoretically also be broken by autoantigens presented on DCs matured during a contemporaneous microbial infection. It seems, though, that the immune system may have yet another level of control to protect against just the kind of scenarios outlined above. In support of this, it was recently reported that the response of conventional human $\mathrm{CD} 4^{+} \mathrm{T}$ cells to autologous peptides presented by mature, but not by immature, DCs results in the generation of regulatory-like $T$ cells (53). If confirmed, this ability of the immune system to so dramatically alter the outcome of a response depending on the antigen being recognized is rather remarkable, especially given the apparently matured DC phenotype. Possibly the net response is attributable to the nature or source of ancillary signals, e.g., which toll-like receptors (TLRs) are being engaged, alone or in combination.

Extrathymically generated regulatory cells represent a heterogeneous assemblage whose ontogenic relationship to naturally occurring Tregs is still being determined. The only really clear point of convergence between the 2 broad families of regulatory cells is that they share a suppressive capability. One interpretation would suggest that peripherally generated regulatory cells merely represent a specialized activation state of conventional $\mathrm{CD} 4^{+}$cells (i.e., "adaptive regulatory cells") whereas $\mathrm{CD} 25^{+} \mathrm{CD} 4^{+}$Tregs are a de facto lineage by virtue of their distinctive Foxp 3 expression, although the most recent data stemming from the use of TGF- $\beta$ or low-zone tolerance induction protocols are perhaps blurring even this distinction $(85,88)$. The use of Foxp3/FOXP3 to disentangle this conundrum has been only partially successful. As far as mice are concerned, Foxp3 seems, by and large, to be a stable marker expressed only in naturally occurring $\mathrm{CD} 25^{+} \mathrm{CD} 4^{+}$Tregs and thus far is not in most models of extrathymically generated regulatory cells (but see refs. 85, 88). In contrast, human FOXP3 expression appears far less stringent, with some reports already demonstrating upregulation in extrathymically generated regulatory cells following even basic activation $(52,53)$. Whether the apparent variability in human FOXP3 and, to a much lesser extent, mouse Foxp3 expression undermines its importance as an unambiguous marker for naturally occurring Tregs remains to be seen.

\section{Conclusion and clinical perspective}

Abundant evidence now strongly supports the once controversial existence of Tregs as key controllers of self tolerance. It also now 
seems that their roles can be expanded to many areas of immunology, in fact, potentially, to any scenario where the suppression and/ or tuning of an immune response is required. A strategic manipulation of Tregs, either naturally occurring or extrathymically generated, to dampen or enhance their functions as appropriate may prove to have great clinical benefit (Table 1 ). Already manipulation of $\mathrm{CD} 25^{+} \mathrm{CD} 4^{+}$Tregs in various animal models has provided encouraging results for both enhancement of tumor immunity and maintenance of allograft tolerance (100-104). In the case of organ transplantation in particular, $\mathrm{CD} 25^{+} \mathrm{CD} 4^{+}$Tregs seem to offer a flexible and adaptive form of immunological control apparently not achievable with standard small-molecule immunosuppression (see for example refs. 102, 104, 105). These practical applications will be expanded upon in other Reviews in this series. Informed by the murine studies, recent experiments are also increasingly demonstrating the significant roles $\mathrm{CD} 25^{+} \mathrm{CD} 4^{+}$Tregs can play in human pathologies as varied as rheumatoid arthritis, multiple sclerosis, HIV infection, and allergy (106-112). Recent advances in our understanding of $\mathrm{CD} 25^{+} \mathrm{CD} 4{ }^{+}$Treg development and important functional markers such as the association with Foxp3/FOXP3 have permitted the accurate isolation and manipulation of these cells in mice and, importantly, their human counterparts. Understanding the events both upstream and downstream of Foxp3/FOXP3 may enable us to "tailor-make" large numbers of $\mathrm{CD} 25^{+} \mathrm{CD} 4^{+}$Tregs to specifically suppress immune responses in autoimmunity and allergy or to antagonize them where a boost of immunity is required, e.g., in microbial and antitumor responses. The potential clinical focus, though, need not be solely on thymically produced $\mathrm{CD} 25^{+} \mathrm{CD} 4^{+}$Tregs, since peripherally generated regulatory cells such as $\operatorname{Tr} 1$, with their potent cytokine-mediated suppressive capacity, may also hold great therapeutic promise.

\section{Acknowledgments}

We apologize to those researchers whose work, because of space restrictions, has not been cited in this review. We thank our colleagues who have allowed the prepublication mention of their work and engaging discussion. Z. Fehérvari is supported by the Japan Society for the Promotion of Science and S. Sakaguchi by grantsin-aid from the Ministry of Education, Culture, Sports, Science and Technology and the Ministry of Human Welfare of Japan.

Address correspondence to: Zoltán Fehérvari, Department of Experimental Pathology, Institute for Frontier Medical Sciences, Kyoto University, Shogo-in 53, Sakyo-ku, Kyoto 606-8507, Japan. Phone: 81-75-751-3888; Fax: 81-75-751-3820; E-mail: zed72@frontier.kyoto-u.ac.jp.
1. Sakaguchi, S. 2004. Naturally arising CD4+ regulatory $\mathrm{t}$ cells for immunologic self-tolerance and negative control of immune responses. Annu. Rev. Immunol. 22:531-562.

2. Bloom, B.R., Salgame, P., and Diamond, B. 1992. Revisiting and revising suppressor T cells. Immunol. Today. 13:131-136.

3. Gambineri, E., Torgerson, T.R., and Ochs, H.D. 2003. Immune dysregulation, polyendocrinopathy, enteropathy, and X-linked inheritance (IPEX), a syndrome of systemic autoimmunity caused by mutations of FOXP3, a critical regulator of T-cell homeostasis. Curr. Opin. Rheumatol. 15:430-435.

4. Roncarolo, M.G., Bacchetta, R., Bordignon, C., Narula, S., and Levings, M.K. 2001. Type 1 T regulatory cells. Immunol. Rev. 182:68-79.

5. Weiner, H.L. 2001. Induction and mechanism of action of transforming growth factor-beta-secreting Th3 regulatory cells. Immunol. Rev. 182:207-214.

6. Bluestone, J.A., and Abbas, A.K. 2003. Natural versus adaptive regulatory T cells. Nat. Rev. Immunol. 3:253-257.

7. Sakaguchi, S., Takahashi, T., and Nishizuka, Y. 1982. Study on cellular events in post-thymectomy autoimmune oophoritis in mice. II. Requirement of Lyt-1 cells in normal female mice for the prevention of oophoritis. J. Exp. Med. 156:1577-1586.

8. Sakaguchi, S., Fukuma, K., Kuribayashi, K., and Masuda, T. 1985. Organ-specific autoimmune diseases induced in mice by elimination of $\mathrm{T}$ cell subset. I. Evidence for the active participation of $\mathrm{T}$ cells in natural self-tolerance; deficit of a $\mathrm{T}$ cell subset as a possible cause of autoimmune disease. J. Exp. Med. 161:72-87.

9. Asano, M., Toda, M., Sakaguchi, N., and Sakaguchi, S. 1996. Autoimmune disease as a consequence of developmental abnormality of a $\mathrm{T}$ cell subpopulation. J. Exp. Med. 184:387-396.

10. Penhale, W.J., Farmer, A., McKenna, R.P., and Irvine, W.J. 1973. Spontaneous thyroiditis in thymectomized and irradiated Wistar rats. Clin. Exp. Immunol. 15:225-236.

11. Fowell, D., and Mason, D. 1993. Evidence that the $\mathrm{T}$ cell repertoire of normal rats contains cells with the potential to cause diabetes. Characterization of the CD4+ T cell subset that inhibits this autoimmune potential. J. Exp. Med. 177:627-636.
12. Boitard, C., Yasunami, R., Dardenne, M., and Bach, J.F. 1989. T cell-mediated inhibition of the transfer of autoimmune diabetes in NOD mice. J. Exp. Med. 169:1669-1680.

13. Greiner, D.L., et al. 1987. Depletion of RT6.1+ T lymphocytes induces diabetes in resistant biobreeding/Worcester (BB/W) rats. J. Exp. Med. 166:461-475.

14. Powrie, F., and Mason, D. 1990. OX-22high CD4+ $T$ cells induce wasting disease with multiple organ pathology: prevention by the OX-22low subset. J. Exp. Med. 172:1701-1708.

15. Powrie, F., Leach, M.W., Mauze, S., Caddle, L.B., and Coffman, R.L. 1993. Phenotypically distinct subsets of CD4+ T cells induce or protect from chronic intestinal inflammation in C. B-17 scid mice. Int. Immunol. 5:1461-1471.

16. Sakaguchi, S., Sakaguchi, N., Asano, M., Itoh, M., and Toda, M. 1995. Immunologic self-tolerance maintained by activated T cells expressing IL-2 receptor alpha-chains (CD25). Breakdown of a single mechanism of self-tolerance causes various autoimmune diseases. J. Immunol. 155:1151-1164.

17. Itoh, M., et al. 1999. Thymus and autoimmunity: production of $\mathrm{CD} 25+\mathrm{CD} 4+$ naturally anergic and suppressive $\mathrm{T}$ cells as a key function of the thymus in maintaining immunologic self-tolerance. J. Immunol. 162:5317-5326.

18. Seddon, B., and Mason, D. 2000. The third function of the thymus. Immunol. Today. 21:95-99.

19. Jordan, M.S., et al. 2001. Thymic selection of $\mathrm{CD} 4+\mathrm{CD} 25+$ regulatory $\mathrm{T}$ cells induced by an agonist self-peptide. Nat. Immunol. 2:301-306.

20. Kawahata, K., et al. 2002. Generation of CD4(+)CD25(+) regulatory $\mathrm{T}$ cells from autoreactive $\mathrm{T}$ cells simultaneously with their negative selection in the thymus and from nonautoreactive $\mathrm{T}$ cells by endogenous TCR expression. J. Immunol. 168:4399-4405.

21. Stephens, G.L., and Ignatowicz, L. 2003. Decreasing the threshold for thymocyte activation biases CD4+ $\mathrm{T}$ cells toward a regulatory $(\mathrm{CD} 4+\mathrm{CD} 25+)$ lineage. Eur. J. Immunol. 33:1282-1291.

22. Sakaguchi, S., et al. 2003. Thymic generation and selection of CD25+CD4+ regulatory T cells: implications of their broad repertoire and high self-reactivity for the maintenance of immunological self- tolerance. Novartis Found. Symp. 252:6-16.

23. Hsieh, C.S., et al. 2004. Recognition of the peripheral self by naturally arising CD25(+) CD $4(+) \mathrm{T}$ cell receptors. Immunity. 21:267-277.

24. Salomon, B., et al. 2000. B7/CD28 costimulation is essential for the homeostasis of the CD4+CD25+ immunoregulatory $\mathrm{T}$ cells that control autoimmune diabetes. Immunity. 12:431-440.

25. Kumanogoh, A., et al. 2001. Increased T cell autoreactivity in the absence of CD40-CD40 ligand interactions: a role of CD40 in regulatory T cell development. J. Immunol. 166:353-360.

26. Lehmann, J., et al. 2002. Expression of the integrin alpha Ebeta 7 identifies unique subsets of CD25+ as well as CD25- regulatory T cells. Proc. Natl. Acad. Sci. U. S. A. 99:13031-13036.

27. Takahashi, T., et al. 2000. Immunologic self-tolerance maintained by $\mathrm{CD} 25(+) \mathrm{CD} 4(+)$ regulatory $\mathrm{T}$ cells constitutively expressing cytotoxic T lymphocyte-associated antigen 4. J. Exp. Med. 192:303-310.

28. Read, S., Malmstrom, V., and Powrie, F. 2000. Cytotoxic T lymphocyte-associated antigen 4 plays an essential role in the function of CD25(+)CD4(+) regulatory cells that control intestinal inflammation. J. Exp. Med. 192:295-302.

29. Shimizu, J., Yamazaki, S., Takahashi, T., Ishida, Y., and Sakaguchi, S. 2002. Stimulation of CD25(+)CD4(+) regulatory T cells through GITreg breaks immunological self-tolerance. Nat. Immunol. 3:135-142.

30. McHugh, R.S., et al. 2002. CD4(+)CD25(+) immunoregulatory $\mathrm{T}$ cells: gene expression analysis reveals a functional role for the glucocorticoidinduced TNF receptor. Immunity. 16:311-323.

31. Bruder, D., et al. 2004. Neuropilin-1: a surface marker of regulatory T cells. Eur. J. Immunol. 34:623-630.

32. Baecher-Allan, C., Brown, J.A., Freeman, G.J., and Hafler, D.A. 2001. CD4+CD25high regulatory cells in human peripheral blood. J. Immunol. 167:1245-1253.

33. Almeida, A.R., Legrand, N., Papiernik, M., and Freitas, A.A. 2002. Homeostasis of peripheral CD4+ T cells: IL-2R alpha and IL-2 shape a population of regulatory cells that controls CD4+ T cell numbers. J. Immunol. 169:4850-4860.

34. Malek, T.R., Yu, A., Vincek, V., Scibelli, P., and Kong, 
L. 2002. CD4 regulatory $\mathrm{T}$ cells prevent lethal autoimmunity in IL-2Rbeta-deficient mice. Implications for the nonredundant function of IL-2. Immunity. 17:167-178.

35. Papiernik, M., de Moraes, M.L., Pontoux, C., Vasseur, F., and Penit, C. 1998. Regulatory CD4 T cells: expression of IL-2R alpha chain, resistance to clonal deletion and IL-2 dependency. Int. Immunol. 10:371-378

36. Kanamaru, F., et al. 2004. Costimulation via glucocorticoid-induced TNF receptor in both conventional and $\mathrm{CD} 25+$ regulatory $\mathrm{CD} 4+\mathrm{T}$ cells J. Immunol. 172:7306-7314.

37. Stephens, G.L. et al. 2004. Engagement of glucocorticoid-induced TNFR family-related receptor on effector $\mathrm{T}$ cells by its ligand mediates resistance to suppression by $\mathrm{CD} 4+\mathrm{CD} 25+\mathrm{T}$ cells. J. Immunol. 173:5008-5020.

38. Tone, M., et al. 2003. Mouse glucocorticoidinduced tumor necrosis factor receptor ligand is costimulatory for T cells. Proc. Natl. Acad. Sci.U. S. A 100:15059-15064.

39. Blair, P.J., et al. 1994. CD4+CD8- T cells are the effector cells in disease pathogenesis in the scurfy (sf) mouse. J. Immunol. 153:3764-3774.

40. Godfrey, V.L., Wilkinson, J.E., Rinchik, E.M., and Russell, L.B. 1991. Fatal lymphoreticular disease in the scurfy ( $\mathrm{sf}$ ) mouse requires $\mathrm{T}$ cells that mature in a sf thymic environment: potential model for thymic education. Proc. Natl. Acad. Sci. U. S. A. 88:5528-5532.

41. Lyon, M.F., Peters, J., Glenister, P.H., Ball, S., and Wright, E. 1990. The scurfy mouse mutant has previously unrecognized hematological abnormalities and resembles Wiskott-Aldrich syndrome. Proc. Natl. Acad. Sci. U. S. A. 87:2433-2437.

42. Brunkow, M.E., et al. 2001. Disruption of a new forkhead/winged-helix protein, scurfin, results in the fatal lymphoproliferative disorder of the scurfy mouse. Nat. Genet. 27:68-73.

43. Bennett, C.L., et al. 2001. The immune dysregulation, polyendocrinopathy, enteropathy, X-linked syndrome (IPEX) is caused by mutations of FOXP3. Nat. Genet. 27:20-21.

44. Schubert, L.A., Jeffery, E., Zhang, Y., Ramsdell, F., and Ziegler, S.F. 2001. Scurfin (FOXP3) acts as a repressor of transcription and regulates $\mathrm{T}$ cell activation. J. Biol. Chem. 276:37672-37679.

45. Khattri, R., Cox, T., Yasayko, S.A., and Ramsdell, F. 2003. An essential role for Scurfin in CD4+CD25+ T regulatory cells. Nat. Immunol. 4:337-342.

46. Hori, S., Nomura, T., and Sakaguchi, S. 2003. Control of regulatory $\mathrm{T}$ cell development by the transcription factor Foxp3. Science. 299:1057-1061.

47. Fontenot, J.D., Gavin, M.A., and Rudensky, A.Y. 2003. Foxp3 programs the development and function of CD4+CD25+ regulatory T cells. Nat. Immunol. 4:330-336.

48. Oswald-Richter, K., et al. 2004. HIV infection of naturally occurring and genetically reprogrammed human regulatory T-cells. PLoS Biol. 2:E198.

49. Weiss, L., et al. 2004. Human immunodeficiency virus-driven expansion of CD4+CD25+ regulatory $\mathrm{T}$ cells which suppress HIV-specific CD4 T-cell responses in HIV-infected patients. Blood. doi:10.1182/blood-2004-01-0365.

50. Walker, M.R., et al. 2003. Induction of FoxP3 and acquisition of $\mathrm{T}$ regulatory activity by stimulated human $\mathrm{CD} 4^{+} \mathrm{CD} 25^{-} \mathrm{T}$ cells. J. Clin. Invest. 112:1437-1443. doi:10.1172/JCI200319441.

51. Yagi, H., et al. 2004. Crucial role of FOXP3 in the development and function of human CD25+CD4+ regulatory $\mathrm{T}$ cells. Int. Immunol. In press.

52. Stassen, M., et al. 2004. Human CD25+ regulatory $T$ cells: two subsets defined by the integrins alpha 4 beta 7 or alpha 4 beta 1 confer distinct suppressive properties upon CD4+ T helper cells. Eur. J. Immunol. 34:1303-1311.
53. Verhasselt, V., et al. 2004. Induction of FOXP3expressing regulatory CD4pos $\mathrm{T}$ cells by human mature autologous dendritic cells. Eur. J. Immunol. 34:762-772

54. Tommasini, A., et al. 2002. X-chromosome inactivation analysis in a female carrier of FOXP3 mutation. Clin. Exp. Immunol. 130:127-130.

55. Maloy, K.J., et al. 2003. CD4+CD25+ T(R) cells suppress innate immune pathology through cytokinedependent mechanisms. J. Exp. Med. 197:111-119.

56. Serra, P., et al. 2003. CD40 ligation releases immature dendritic cells from the control of regulatory CD4+CD25+ T cells. Immunity. 19:877-889.

57. Cederbom, L., Hall, H., and Ivars, F. 2000. $\mathrm{CD} 4+\mathrm{CD} 25+$ regulatory $\mathrm{T}$ cells down-regulate costimulatory molecules on antigen-presenting cells. Eur. J. Immunol. 30:1538-1543.

58. Takahashi, T., et al. 1998. Immunologic self-tolerance maintained by $\mathrm{CD} 25+\mathrm{CD} 4+$ naturally anergic and suppressive $\mathrm{T}$ cells: induction of autoimmune disease by breaking their anergic/suppressive state. Int. Immunol. 10:1969-1980.

59. Thornton, A.M., and Shevach, E.M. 1998. CD4+CD25+ immunoregulatory $\mathrm{T}$ cells suppress polyclonal $\mathrm{T}$ cell activation in vitro by inhibiting interleukin 2 production. J. Exp. Med. 188:287-296.

60. Janssens, W., Carlier, V., Wu, B., VanderElst, L., Jacquemin, M.G., and Saint-Remy, J.M. 2003. CD4+CD25+ T cells lyse antigen-presenting B cells by Fas-Fas ligand interaction in an epitope-specific manner. J. Immunol. 171:4604-4612.

61. Thornton, A.M., Donovan, E.E., Piccirillo, C.A., and Shevach, E.M. 2004. Cutting edge: IL-2 is critically required for the in vitro activation of CD4+CD25+ T cell suppressor function. J. Immunol. 172:6519-6523.

62. Thornton, A.M., Piccirillo, C.A., and Shevach, E.M 2004. Activation requirements for the induction of CD4+CD25+ T cell suppressor function. Eur. J. Immunol. 34:366-376.

63. Yamazaki, S., et al. 2003. Direct expansion of functional CD25+CD4+ regulatory $\mathrm{T}$ cells by antigen-processing dendritic cells. J. Exp. Med. 198:235-247.

64. Pasare, C., and Medzhitov, R. 2003. Toll pathway-dependent blockade of CD4+CD25+ T cellmediated suppression by dendritic cells. Science. 299:1033-1036.

65. Gavin, M.A., Clarke, S.R., Negrou, E., Gallegos, A., and Rudensky, A. 2002. Homeostasis and anergy of CD4(+)CD25(+) suppressor T cells in vivo. Nat. Immunol. 3:33-41.

66. Asseman, C., Mauze, S., Leach, M.W., Coffman, R.L., and Powrie, F. 1999. An essential role for interleukin 10 in the function of regulatory $\mathrm{T}$ cells that inhibit intestinal inflammation. J. Exp. Med. 190:995-1004

67. Suri-Payer, E., and Cantor, H. 2001. Differential cytokine requirements for regulation of autoimmune gastritis and colitis by CD4(+)CD25(+) T cells. J. Autoimmun. 16:115-123.

68. Berg, D.J., et al. 1996. Enterocolitis and colon cancer in interleukin-10-deficient mice are associated with aberrant cytokine production and CD4(+) TH1-like responses. J. Clin. Invest. 98:1010-1020.

69. Powrie, F., Carlino, J., Leach, M.W., Mauze, S., and Coffman, R.L. 1996. A critical role for transforming growth factor-beta but not interleukin 4 in the suppression of T helper type 1-mediated colitis by CD45RB(low) CD4+ T cells. J. Exp. Med. 183:2669-2674.

70. Nakamura, K., Kitani, A., and Strober, W. 2001. Cell contact-dependent immunosuppression by $\mathrm{CD} 4(+) \mathrm{CD} 25(+)$ regulatory $\mathrm{T}$ cells is mediated by cell surface-bound transforming growth factor beta. J. Exp. Med. 194:629-644.

71. Oida, T., et al. 2003. CD4+CD25- T cells that express latency-associated peptide on the surface suppress CD4+CD45RBhigh-induced colitis by a TGF-beta-dependent mechanism. J. Immunol. 170:2516-2522.

72. Piccirillo, C.A., et al. 2002. CD4(+)CD25(+) regulatory $\mathrm{T}$ cells can mediate suppressor function in the absence of transforming growth factor beta 1 production and responsiveness. J. Exp. Med. 196:237-246

73. Zhang, X., Izikson, L., Liu, L., and Weiner, H.L. 2001. Activation of CD25(+)CD4(+) regulatory T cells by oral antigen administration. J. Immunol. 167:4245-4253

74. De La Rosa, M., Rutz, S., Dorninger, H., and Scheffold, A. 2004. Interleukin-2 is essential for CD4(+)CD25(+) regulatory T cell function. Eur. J. Immunol. 34:2480-2488.

75. Grohmann, U., et al. 2002. CTLA-4-Ig regulates tryptophan catabolism in vivo. Nat. Immunol. 3:1097-1101.

76. Fallarino, F., et al. 2003. Modulation of tryptophan catabolism by regulatory $\mathrm{T}$ cells. Nat. Immunol. 4:1206-1212.

77. Munn, D.H., Sharma, M.D., and Mellor, A.L. 2004. Ligation of B7-1/B7-2 by human CD4(+) T cells triggers indoleamine 2,3-dioxygenase activity in dendritic cells. J. Immunol. 172:4100-4110.

78. Collins, A.V., et al. 2002. The interaction properties of costimulatory molecules revisited. Immunity. 17:201-210.

79. Piccirillo, C.A., and Shevach, E.M. 2001. Cutting edge: control of CD8 $+\mathrm{T}$ cell activation by CD4+CD25+ immunoregulatory cells. J. Immunol. 167:1137-1140

80. Paust, S., Lu, L., McCarty, N., and Cantor, H. 2004. Engagement of B7 on effector $\mathrm{T}$ cells by regulatory $\mathrm{T}$ cells prevents autoimmune disease. Proc. Natl. Acad. Sci. U. S. A. 101:10398-10403.

81. Greenfield, E.A., et al. 1997. B7.2 expressed by T cells does not induce CD28-mediated costimulatory activity but retains CTLA4 binding: implications for induction of antitumor immunity to $\mathrm{T}$ cell tumors. J. Immunol. 158:2025-2034.

82. Prabhu Das, M.R., et al. 1995. Reciprocal expression of co-stimulatory molecules, B7-1 and B7-2, on murine $\mathrm{T}$ cells following activation. Eur. J. Immunol. 25:207-211.

83. Workman, C.J., and Vignali, D.A. 2003. The CD4related molecule, LAG-3 (CD223), regulates the expansion of activated T cells. Eur. J. Immunol. 33:970-979.

84. Huang, C.-T., et al. Role of LAG-3 in regulatory $\mathrm{T}$ cells. Immunity. In press.

85. Apostolou, I., and Von Boehmer, H. 2004. In vivo instruction of suppressor commitment in naive $\mathrm{T}$ cells. J. Exp. Med. 199:1401-1408.

86. Mitchison, N.A. 1964. Induction of immunological paralysis in two zones of dosage. Proc. R. Soc. Lond. B Biol. Sci. 161:275-292.

87. Gorelik, L., and Flavell, R.A. 2002. Transforming growth factor-beta in T-cell biology. Nat. Rev. Immunol. 2:46-53.

88. Chen, W., et al. 2003. Conversion of peripheral CD4+CD25- naive $\mathrm{T}$ cells to $\mathrm{CD} 4+\mathrm{CD} 25+$ regulatory $\mathrm{T}$ cells by TGF-beta induction of transcription factor Foxp3. J. Exp. Med. 198:1875-1886.

89. Groux, H., et al. 1997. A CD4+ T-cell subset inhibits antigen-specific T-cell responses and prevents colitis. Nature. 389:737-742.

90. Chen, Y., Kuchroo, V.K., Inobe, J., Hafler, D.A., and Weiner, H.L. 1994. Regulatory T cell clones induced by oral tolerance: suppression of autoimmune encephalomyelitis. Science. 265:1237-1240.

91. Groux, H. 2003. Type 1 T-regulatory cells: their role in the control of immune responses. Transplantation. 75(Suppl. 9):8S-12S

92. Barrat, F.J., et al. 2002. In vitro generation of interleukin 10-producing regulatory CD4(+) T cells is induced by immunosuppressive drugs and 
inhibited by T helper type 1 (Th1)- and Th2-inducing cytokines. J. Exp. Med. 195:603-616.

93. Vieira, P.L., et al. 2004. IL-10-secreting regulatory T cells do not express Foxp3 but have comparable regulatory function to naturally occurring CD4+CD25+ regulatory T cells. J. Immunol. 172:5986-5993.

94. Gregori, S., et al. 2001. Regulatory T cells induced by 1 alpha,25-dihydroxyvitamin D3 and mycophenolate mofetil treatment mediate transplantation tolerance. J. Immunol. 167:1945-1953.

95. Kemper, C., et al. 2003. Activation of human CD4+ cells with CD3 and CD46 induces a T-regulatory cell 1 phenotype. Nature. 421:388-392.

96. Sato, K., Yamashita, N., Baba, M., and Matsuyama, T. 2003. Regulatory dendritic cells protect mice from murine acute graft-versus-host disease and leukemia relapse. Immunity. 18:367-379.

97. Sato, K., Yamashita, N., Baba, M., and Matsuyama, T. 2003. Modified myeloid dendritic cells act as regulatory dendritic cells to induce anergic and regulatory T cells. Blood. 101:3581-3589.

98. Jonuleit, H., Schmitt, E., Schuler, G., Knop, J., and Enk, A.H. 2000. Induction of interleukin 10-producing, nonproliferating CD4(+) T cells with regulatory properties by repetitive stimulation with allogeneic immature human dendritic cells. J. Exp. Med. 192:1213-1222.

99. Steinman, R.M., Hawiger, D., and Nussenzweig, M.C. 2003. Tolerogenic dendritic cells. Annu. Rev. Immunol. 21:685-711.
100.Shimizu, J., Yamazaki, S., and Sakaguchi, S. 1999. Induction of tumor immunity by removing CD25+CD4+ T cells: a common basis between tumor immunity and autoimmunity. J. Immunol. 163:5211-5218.

101.Sutmuller, R.P., et al. 2001. Synergism of cytotoxic $\mathrm{T}$ lymphocyte-associated antigen 4 blockade and depletion of CD25(+) regulatory $\mathrm{T}$ cells in antitumor therapy reveals alternative pathways for suppression of autoreactive cytotoxic T lymphocyte responses. J. Exp. Med. 194:823-832.

102.Edinger, M., et al. 2003. CD4+CD25+ regulatory $\mathrm{T}$ cells preserve graft-versus-tumor activity while inhibiting graft-versus-host disease after bone marrow transplantation. Nat. Med. 9:1144-1150.

103.Trenado, A., et al. 2003. Recipient-type specific $\mathrm{CD} 4{ }^{+} \mathrm{CD} 25^{+}$regulatory $\mathrm{T}$ cells favor immune reconstitution and control graft-versus-host disease while maintaining graft-versus-leukemia. J. Clin. Invest. 112:1688-1696. doi:10.1172/ JCI200317702.

104.Nishimura, E., Sakihama, T., Setoguchi, R., Tanaka, K., and Sakaguchi, S. 2004. Induction of antigenspecific immunologic tolerance by in vivo and in vitro antigen-specific expansion of naturally arising Foxp3+CD25+CD4+ regulatory T cells. Int. Immunol. 16:1189-1201.

105.Hoffmann, P., Ermann, J., Edinger, M., Fathman, C.G., and Strober, S. 2002. Donor-type $\mathrm{CD} 4(+) \mathrm{CD} 25(+)$ regulatory $\mathrm{T}$ cells suppress lethal acute graft-versus-host disease after allogeneic bone marrow transplantation. J. Exp. Med. 196:389-399.

106. Baecher-Allan, C., and Hafler, D.A. 2004. Suppressor T cells in human diseases. J. Exp. Med. 200:273-276.

107.Viglietta, V., Baecher-Allan, C., Weiner, H.L., and Hafler, D.A. 2004. Loss of functional suppression by CD 4+CD25+ regulatory $\mathrm{T}$ cells in patients with multiple sclerosis. J. Exp. Med. 199:971-979.

108.Karlsson, M.R., Rugtveit, J., and Brandtzaeg, P. 2004. Allergen-responsive CD4+CD25+ regulatory $\mathrm{T}$ cells in children who have outgrown cow's milk allergy. J. Exp. Med. 199:1679-1688.

109. Ehrenstein, M.R., et al. 2004. Compromised function of regulatory $\mathrm{T}$ cells in rheumatoid arthritis and reversal by anti-TNF $\alpha$ therapy. J. Exp. Med. 200:277-285

110.Kinter, A.L., et al. 2004. CD25+CD4+ regulatory T cells from the peripheral blood of asymptomatic $\mathrm{HIV}$-infected individuals regulate CD4+ and CD8+ HIV-specific T cell immune responses in vitro and are associated with favorable clinical markers of disease status. J. Exp. Med. 200:331-343.

111. Cao, D., et al. 2003. Isolation and functional characterization of regulatory CD25brightCD4+ T cells from the target organ of patients with rheumatoid arthritis. Eur. J. Immunol. 33:215-223.

112.Cao, D., van Vollenhoven, R., Klareskog, L., Trollmo, C., and Malmstrom, V. 2004. CD25brightCD4+ regulatory $\mathrm{T}$ cells are enriched in inflamed joints of patients with chronic rheumatic disease. Artbritis Res. Ther. 6:R335-R346. 